\title{
Arzt und Kulturvermittler
}

\section{Matthias Scholer}

Online- und Printredaktor SÄZ

\author{
Rund ein Drittel der Ärztinnen und Ärzte in der Schweiz kommt aus dem Ausland. \\ Weshalb haben sie sich für ein Leben hierzulande entschieden? Welches sind kul- \\ turelle Stolpersteine, die es zu umschiffen gilt? Und was halten sie von der Schwei- \\ zer Gesundheitspolitik? Diesen und weiteren Fragen möchten wir in der neuen, in \\ loser Folge erscheinenden Artikelserie «Grüezi Schweiz» nachgehen. In dieser Aus- \\ gabe stellen wir Mohammed Taha vor, der sich mit seiner Niederlassung und der \\ Übernahme einer Hausarztpraxis in der Schweiz seinen Lebenstraum erfüllen \\ konnte.
}

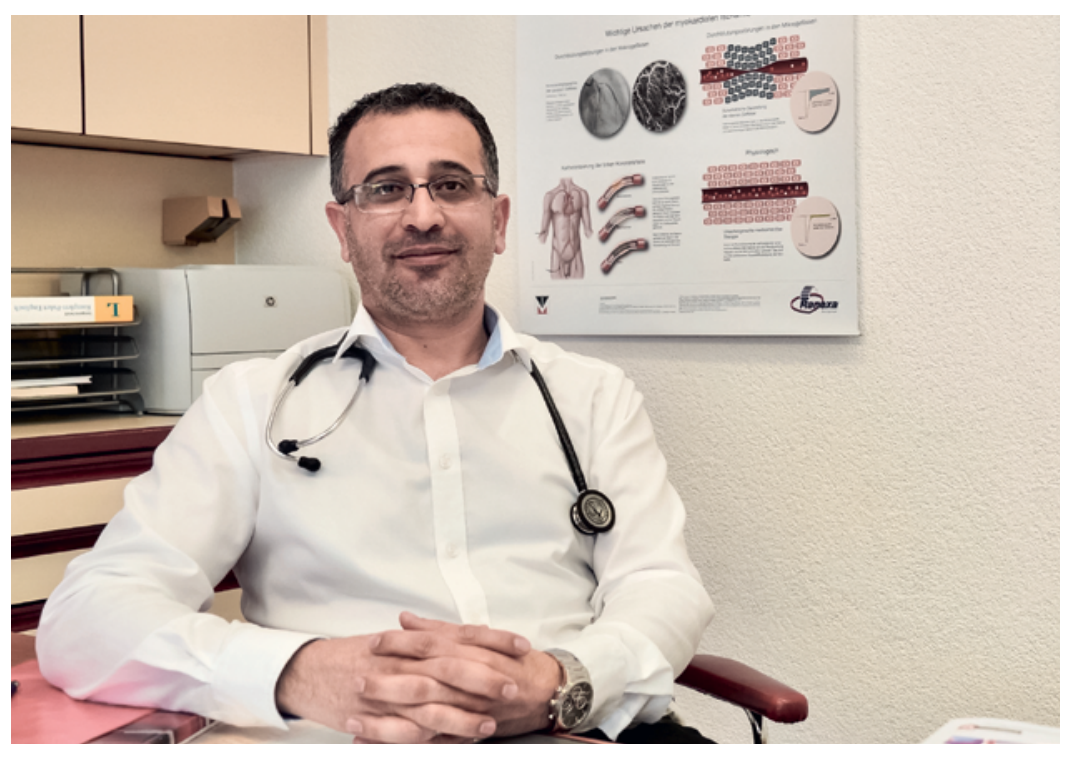

Mohammed Taha ist glücklich, in der Schweiz arbeiten und leben zu dürfen.

Gazastreifen 1995. Mohammed Taha muss in der Schule einen Vortrag über die Schweiz halten. Da es zu dieser Zeit noch kaum Internet gibt, informiert sich der Schüler in Büchern und Zeitschriften über das ferne Land. «Dabei verliebte ich mich in die Schweiz», erzählt Mohammed Taha. Insbesondere die Berichte über die gut ausgebaute Infrastruktur, die schöne Natur und das politische System, welches eine direkte Demokratie erlaubt, faszinierten den jungen Palästinenser nachhaltig. "Ich träumte seither von einem Leben in der Schweiz», sagt Mohammed Taha. Doch dies war nicht sein einziger Traum. Taha wollte seit frühster Kindheit Medizin studieren. Ein Medizinstudium und ein Leben in der Schweiz - ein äusserst ambitioniertes Ziel für einen in Gaza lebender Sohn eines Lehrers.

\section{Harziger Start}

Die erste Enttäuschung liess nicht lange auf sich warten. Als Taha von den hohen Lebenskosten in der Schweiz erfuhr, stand fest, dass ein Studium in seinem Traumland niemals in Frage kommen würde. «Für mich brach eine Welt zusammen", erinnert er sich. Erst als ihm ein Arzt in Palästina von der Möglichkeit eines Studiums in Deutschland erzählte, schöpfte Mohammed Taha wieder Hoffnung. Seine Eltern gaben ihm ihre gesamten Ersparnisse, und so begann ein eindrücklicher Werdegang. Taha reiste nach Berlin, lernte dort während einem Jahr Deutsch und bereitete sich auf sein Studium vor, das er dann in Köln absolvierte. «Das war eine äusserst harte Zeit. Ich war völlig auf mich gestellt und hatte kaum Geld, da das Studium zu intensiv war, um daneben noch einem Job nachzugehen", erzählt Taha.

\section{Über Umwege in die Schweiz}

Nach dem Staatsexamen durchlief der Palästinenser in Deutschland verschiedene Stationen, bis er schlussendlich seine Facharztprüfung in Innerer Medizin absolvieren konnte. Damit war ein Teil seines ursprünglichen Traums erfüllt. Als Mohammed Taha während eines Besuchs in seinem Heimatland seine heutige Frau kennenlernte, entschloss er sich, die nächste Herausforderung anzugehen - den Umzug in die Schweiz. "Auf eine meiner Blindbewerbungen antwortete das 
Spital Rheinfelden», berichtet der Arzt. Trotz seiner bestandenen Facharztprüfung musste sich hier Taha mit einer Stelle als Assistenzarzt zufriedengeben. Aber: «Ich war so glücklich, in meinem Traumland arbeiten zu dürfen.» Vor allem die Verlässlichkeit und der Umgang zwischen den Berufskolleginnen und -kollegen, aber auch mit den Patienten beeindruckten Taha sehr. Doch mit etwas hatte er zu kämpfen: «Ich verstand fast nichts, wenn die Personen im Dialekt sprachen.» Zwar wechselten die meisten Patienten ins Hochdeutsche, doch der Sprachwechsel behinderte häufig den Redefluss, worunter das Arzt-Patienten-Gespräch litt. Für Mohammed Taha stand deshalb fest: «Ich musste den Dialekt lernen.» Dabei half ihm insbesondere Radiohören. Heute versteht der gebürtige Palästinenser fast alle seiner Patienten. Er selber spricht lieber Hochdeutsch, dies aber fliessend.

\section{Hausarzt aus Leidenschaft}

Nach verschiedenen Stellen in der Schweiz wusste Taha: «Mich faszinierte die Herausforderung der Diagnosestellung, die persönliche Beratung der Patientinnen und Patienten und die Abwechslung im ambulanten Bereich.» So packte der Mediziner eine weitere Chance, die ihm das Schicksal bot: «Meine Frau erwartete unser erstes Kind, und die Hebamme erzählte uns von einer Hausarztpraxis in Stein, für welche seit längerem eine Nachfolgeregelung gesucht wurde.» Nach reiflicher Überlegung entschloss sich Mohammed Taha, diese Praxis 2015 zu übernehmen, auch wenn das Geld dafür knapp war. «Ich konnte genügend Geld ansparen, um sechs Monate ohne grosses Einkommen zu überbrücken», sagt der Hausarzt. Diese Zeit war nicht einfach: "Ich hatte im ersten Halbjahr vielleicht drei bis vier Patienten am Tag.» Und er fügt lächelnd an: «Mohammed ist vielleicht auch nicht gerade der einfachste Vorname, um in einer kleinen Gemeinde Fuss zu fassen.» Für seine zu Beginn wenigen Patienten nahm sich Taha dafür sehr viel Zeit. Und dies sprach sich herum. Die Mundpropaganda begann zu wirken. Immer mehr Patientinnen und Patienten suchten seine Praxis auf. Ein entscheidender Tipp, wie er die noch bestehenden Berührungsängste weiter abbauen konnte, kam von einer Schweizer Patientin. «Sie riet mir, meine Arztdiplome im Wartezimmer aufzuhängen.» Denn es gingen die Gerüchte um, dass er gar kein richtiger Arzt sei. «Und dies obwohl ich bei den Krankenkassen gelistet war und über diese seit dem ersten Tag abrechnete», erzählt Mohammed Taha schmunzelnd. Und noch etwas lernte er von seinen Schweizer Patienten: ein kräftiger Händedruck bei der Begrüssung und Verabschiedung ist wichtig.

\section{Nicht nur eitel Sonnenschein}

Heute setzt sich seine Patientenpopulation hälftig aus Einheimischen und Ausländern zusammen. Insbesondere arabisch sprechende Mitmenschen wenden sich gerne an Mohammed Taha, da sie mit ihm ohne Sprachbarriere kommunizieren können und er beide Kulturen kennt. Der Palästinenser sieht sich denn auch als Arzt und Kulturvermittler: «Die Immigranten wissen nicht, wie unser Gesundheitssystem funktioniert.» Wann man in eine Notfallstation geht und wann zum Hausarzt oder Apotheker, dass man für einen Arztbesuch einen Termin braucht und auf der anderen Seite Termine dann auch wahrnimmt, versucht Mohammed Taha mit viel Fingerspitzengefühl seinen Patienten beizubringen. Der Mediziner betont: «Die meisten Fehler werden aus Unwissenheit begangen.»

Doch auch in Stein und in Tahas Leben ist nicht alles eitel Sonnenschein. Letztes Jahr wurde er von einem Schweizer im Zug aufs Übelste beschimpft und bedroht - und dies nur, weil er nicht wie ein Sohn Wilhelm Tells aussieht. Obwohl viele Passagiere die bedrohliche Situation mitverfolgten, schritt niemand schlichtend ein oder verständigte die Polizei. Mohammed Taha musste schlussendlich in einem günstigen Augenblick aus dem Zug flüchten. "Solche Erlebnisse schmerzen", sagt der Hausarzt. Und dennoch: Für Taha ist mit der Niederlassung und der ärztlichen Tätigkeit in der Schweiz sein Lebenstraum in Erfüllung gegangen, den er hart erkämpfen musste. Oder wie es Mohammed Taha ausdrückt: «Ende gut, alles gut.»

Bildnachweis

Matthias Scholer

Für unsere Serie "Grüezi Schweiz» suchen wir ausländische Ärztinnen und Ärzte, die uns einen Einblick in ihr Leben und ihren Berufsalltag gewähren. Wir freuen uns über eine Kontaktaufnahme: mscholer[at]emh.ch 